\title{
Floating aortic thrombus in a patient with non-Hodgkin's lymphoma
}

\author{
Ayşegül Öz Aksu, Figen Başaran Demirkazık
}

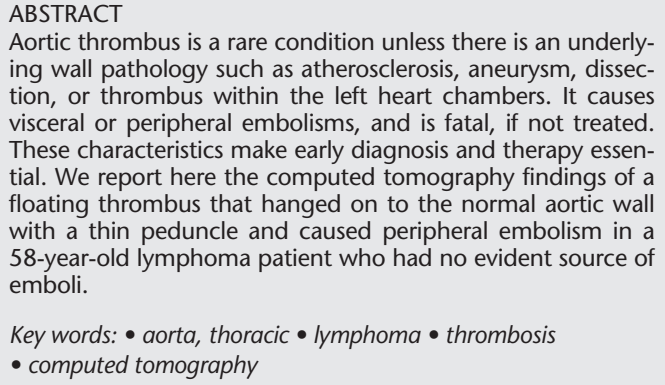

Aortic thrombus is a rare condition unless there is an underlying wall pathology such as atherosclerosis, aneurysm, dissection, or thrombus within the left heart chambers. It causes visceral or peripheral embolisms, and is fatal, if not treated. These characteristics make early diagnosis and therapy essential. We report here the computed tomography findings of a floating thrombus that hanged on to the normal aortic wall with a thin peduncle and caused peripheral embolism in a 58-year-old lymphoma patient who had no evident source of emboli.

Key words: $\bullet$ aorta, thoracic $\bullet$ lymphoma $\bullet$ thrombosis - computed tomography

From the Department of Radiology (A.Ö.A. $₫$ aozaksu@ hacettepe.edu.tr), Hacettepe University School of Medicine, Ankara, Turkey.

Received 25 July 2007; revision requested 25 February 2008; revision received 5 March 2008; accepted 11 March 2008.

Published online 5 October 2009

DOI 10.4261/1305-3825.DIR.1226-07.1
O nly few cases of aortic thrombus in the absence of atherosclerosis, dissection or aneurysm have been reported in the literature. Aortic thrombus is an uncommon condition even in hypercoagulability states like sepsis, polycytemia, disseminated intravascular coagulation, autoimmune diseases, pregnancy, and cancer (1). We report here computed tomography (CT) findings of a floating aortic thrombus that caused peripheral embolism in a B-cell non-Hodgkin lymphoma patient in the absence of any other predisposing factors.

\section{Case report}

An ulcerovegetating mass in the stomach was detected in the upper gastrointestinal endoscopy of a 58-year-old man who had epigastric pain, abdominal distention, weight loss, night sweat and fever complaints. Biopsy revealed CD20 positive, B-cell non-Hodgkin lymphoma.

For staging, thoracoabdominal CT was obtained with a 4-channel multidetector CT scanner with a $5 \mathrm{~mm}$ slice thickness after i.v. injection of $150 \mathrm{~mL}$ of iohexol at a rate of $3.5 \mathrm{~mL} / \mathrm{s}$. For thoracic CT the delay time was $25 \mathrm{~s}$, and for abdominal CT the delay time was $60 \mathrm{~s}$. On thoracic CT images, enlarged left supraclavicular, subcarinal, right paracardiac, bilateral internal mammary lymph nodes, thymus invasion, and bilateral pleural effusions were observed. Additionally, a floating thrombus originating from the aortic arch and extending into the descending thoracic aorta was detected. The thrombus was causing partial obstruction of the descending thoracic aorta lumen (Fig. 1). On sagittal reformatted images, it was observed that the thrombus clinged to the aortic wall with a very thin peduncle (Fig. 2). There were no signs of atherosclerosis, aneurysm, dissection, or cardiac thrombus. On abdominal CT scans, a retroperitoneal mass on the left, massive ascites, mesenteric lymphadenopathies and a wedge shaped splenic infarct were observed.

Chemotherapy and anticoagulant therapy were immediately started after CT examination. At the 36th hour of chemotherapy and anticoagulant therapy, acute and progressive deterioration of liver and renal function tests and elevation of cardiac biomarkers were detected. Abdominal distension markedly increased, unconsciousness ensued, and peripheral circulation failed. Death occurred at the 40th hour of treatment.

\section{Discussion}

Aortic thrombus may lead to catastrophic consequences as a result of acute or chronic recurrent cerebrovascular, coronary, visceral and peripheral embolism. The mortality rate can be as high as $50 \%$, if not treated. Embolic complications can usually be prevented with early diagnosis and treatment $(1,2)$. At least $8-65 \%$ of aortic emboli originate from the left atrium secondary to atrial fibrillation. Primary thrombus 

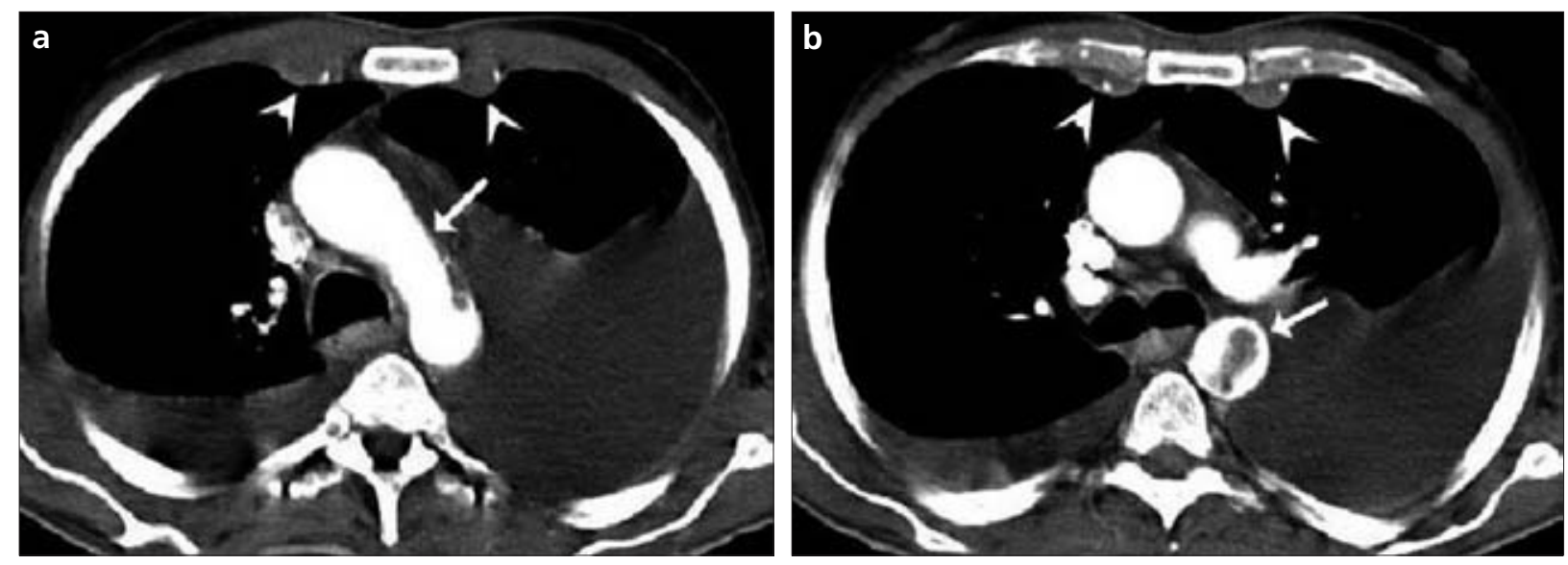

Figure 1. a, b. Post-contrast transverse CT images show the filling defect within the proximal segment of the descending thoracic aorta (arrows) secondary to a free-floating thrombus. The thrombus appears to be attached to the anterior wall of the aortic arch (arrow, a). Note bilateral lymphadenopathy (arrowheads) and pleural effusions.

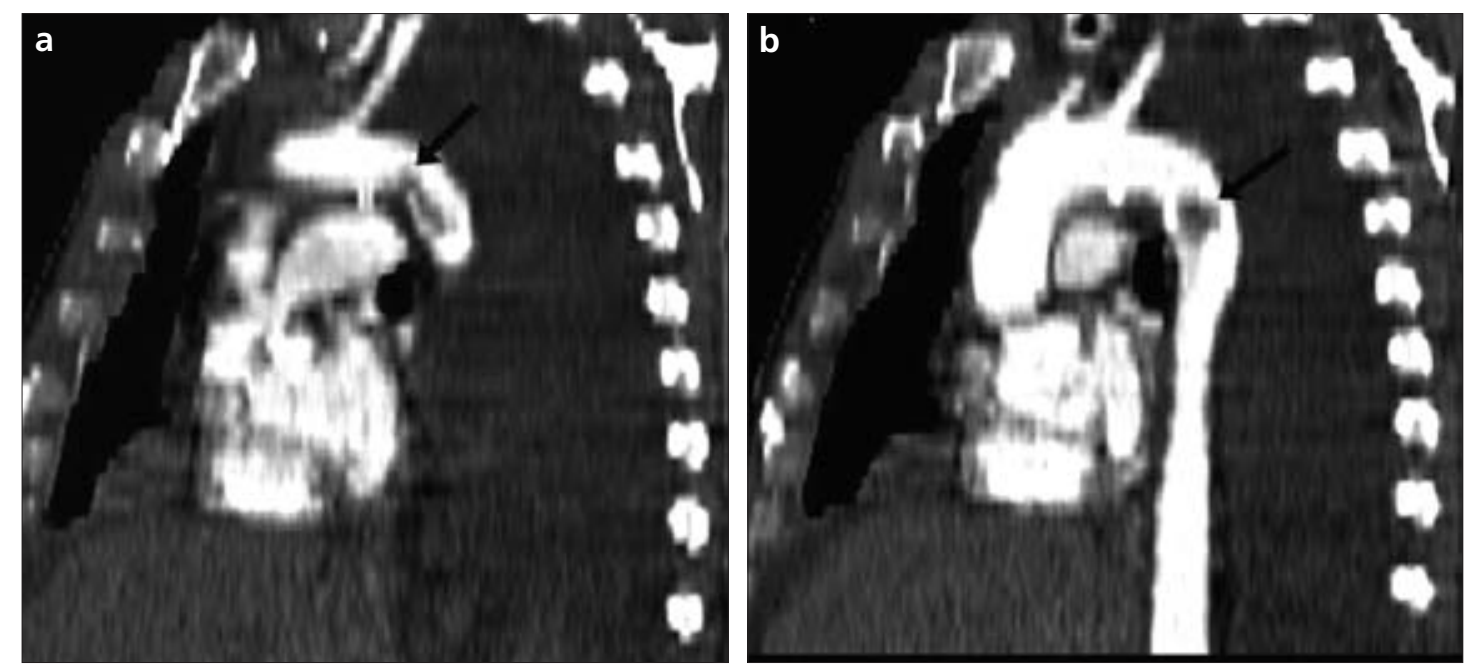

Figure 2. a, b. Sagittal reformatted CT images demonstrate that the floating thrombus is attached to the anterior wall of the aortic arch with a thin peduncle and extended into the descending aorta lumen (arrows).

accounts for the remaining 35-92\%, and most of the cases occur in the setting of underlying atherosclerosis, aneurysm or dissection (3). In young patients, hypercoagulability states like sepsis, polycytemia, disseminated intravascular coagulation, antiphospolipid syndrome, and protein $\mathrm{S}$ deficiency may precipitate thrombosis of the aorta (4-7).

Although hypercoagulation and venous thrombosis are well known, arterial thrombus is found to be very infrequent in cancer patients. In the literature, a few cases of aortic thrombus, which occurred in acute leukemia, pancreatic adenocarcinoma and T-cell lymphoma patients, are reported. Usually, these cases are treated medically with anticoagulant therapy $(1,8)$.

In the reported case no autopsy was performed; nonetheless, we strongly suspect that the patient had developed massive thromboembolism based on secondary findings, such as elevated liver enzymes and abdominal distension that may indicate a mesenteric vascular event, deterioration of renal functions that may point to a renal vascular occlusion, elevated cardiac biomarkers that may suggest coronary arterial embolism, and unconsciousness that can be the consequence of cerebrovascular embolism. Likewise, the splenic infarct detected on the pretreatment CT probably occurred as a result of embolism (9).

Treatment options of the floating aortic thrombus can be summarized as anticoagulation, thrombectomy, endarterectomy, and graft implantation. Since the ideal treatment of mobile aortic thrombi without atheromatosis has not been ascertained, the imme- diate treatment is usually selected according to the underlying pathology and patients' comorbidities. It is also emphasized that surgery should always be considered in the absence of risk factors for surgery (e.g., atherosclerosis), especially if systemic embolism occurs $(10,11)$.

Cross-sectional imaging techniques like MDCT provide fast and noninvasive detection of aortic thrombi. MDCT can also be recommended for close follow-up after medical or surgical therapy.

In conclusion, this case demonstrates an aortic pedunculated free-floating thrombus in a B-cell non-Hodgkin lymphoma patient, which might have caused massive visceral and peripheral embolism, in the absence of underlying atherosclerosis, aneurysm, dissection, or cardiac disorders. 


\section{References}

1. Poiree S, Monnier-Cholley L, Tubiana JM, Arrive L. Acute abdominal aortic thrombosis in cancer patients. Abdom Imaging 2004; 29:511-513.

2. Braunwald B, Libby Z. Braunwald's heart disease. 7th ed. 2005; 1431.

3. Babu SC, Shah PM, Nitahara J. Acute aortic occlusion: factors that influence outcome. J Vasc Surg 1995; 21:567-575.

4. Towne JB. Hypercoagulable states and unexplained vasculer thrombosis. 3rd ed. St.Louis: Quality Medical Publishing. 1991; 101-118.

5. Insko EK, Haksal ZJ. Antiphospholipid syndrome: patterns of life-threatening and severe recurrent vasculer complication. Radiology 1997; 202:319-326.
6. Hazirolan T, Perler BA, Bluemke DA. Floating thoracic aortic thrombus in "protein S" deficient patient. J Vasc Surg 2004; 40:381.

7. Grothues F, Welte T, Grote HJ, Roessner A, Klein HU. Floating aortic thrombus in systemic aspergillosis and detection by transesophageal echocardiography. Crit Care Med 2002; 30:2355-2358.

8. Leong KW, Bosco JJ, Shaik IB. Acute myelomonocytic leukaemia complicated by an acute aortic thrombosis. Postgrad Med J 1995; 71:112-113.

9. Bruno P, Massetti M, Babatasi G, Khayat A. Catastrophic consequences of a free floating thrombus in ascending aorta. Eur Cardiothorac Surg 2001; 19:99-101.
10. Kalangos A, Baldovinos A, Vuille C, Montessuit M, Faidutti B. Floating thrombus in the ascending aorta: a rare cause of peripheral emboli. J Vasc Surg 1997; 26:150-154.

11. Stöllberger C, Kopsa W, Finsterer J. Resolution of an aortic thrombus under anticoagulant therapy. Eur J Cardiothorac Surg 2001; 20:880-882. 